\title{
Ultrasonic cavity solitons
}

\author{
I. Pérez-Arjona ${ }^{1}$, V. J. Sánchez-Morcillo ${ }^{1}$, and G. J. de Valcárcel ${ }^{2}$ \\ ${ }^{1}$ Departament de Física Aplicada, Universitat Politècnica de València, \\ Crta. Natzaret-Oliva s/n, 46730 Grau de Gandia, Spain and \\ ${ }^{2}$ Departament d’Òptica, Universitat de València, Dr. Moliner 50, 46100-Burjassot, Spain
}

\begin{abstract}
We report on a new type of localized structure, an ultrasonic cavity soliton, supported by large aspect-ratio acoustic resonators containing viscous media. The spatio-temporal dynamics of this system is analyzed on the basis of a generalized Swift-Hohenberg equation, derived from the microscopic equations under conditions close to nascent bistability. These states of the acoustic and thermal fields are robust structures, existing whenever a spatially uniform solution and a periodic pattern coexist. An analytical solution for the ultrasonic cavity soliton is also presented.

PACS: 05.45.-a, 75.80.+q, 43.25.+y
\end{abstract}

Many systems in nature, when driven far from equilibrium, can self-organize giving rise to a large variety of patterns or structures. Although studied intensively for most of the last century, it has only been during the past thirty years that pattern formation has emerged as an own branch of science [1]. One of the most relevant features of pattern formation is its universality: systems with different microscopic descriptions frequently exhibit similar patterns on a macroscopic level. This universal character of pattern formation is evidenced when the microscopic models are reduced, under given assumptions, to simpler equations describing the evolution of a single variable, the so called order parameter [2]. Order parameter equations are usually obtained near some critical point, and belong to few and well known classes, such as the Ginzburg-Landau or the Swift-Hohenberg equations and their variants. They are often based on system symmetries and are independent of the microscopic differences among systems, providing a theoretical framework to understand the origins of non-equilibrium pattern formation [1].

This approach to pattern formation has been extensively applied to nonlinear optical cavities [3, 4, 5], such as lasers, optical parametric oscillators or Kerr (cubically nonlinear) resonators, where light in the transverse plane of the cavity has been shown to develop patterns with different symmetries (rolls, hexagons, and also quasipatterns) as well as cavity solitons (CS). The latter correspond to localized solutions often resulting from a bistability between two stationary, spatially extended states of the system, in the form of either self-trapped switching waves between two homogeneous states, or isolated pattern elements embedded in a homogeneous background. Optical CSs are particularly interesting objects because of their potential use as memory bits in optical information processing systems [6]. However, despite the existing analogies between optics and acoustics, acoustical resonators in the nonlinear regime have been much less explored than their optical counterparts. Furthermore, pattern formation studies in acoustics are almost lacking. The main reason lies in the weak dispersion of sound in common homogeneous media, which is responsible for the growth of higher harmonics, leading to wave distortion and shock formation. These effects are absent in optics, which is dispersive in nature. However, in some special cases it is still possible to avoid the nonlinear distortion and recover the analogies [7]. It is, for example, the case of sound beams propagating in viscous media characterized by a strong absorption (e.g. glycerine), where sound velocity depends of fluid temperature, resulting in an additional nonlinearity mechanism of thermal origin. For the majority of fluids, temperature variations induced by an intense acoustic field result in a decrease of sound velocity, leading to a self-focusing of the beam. In the case of viscous fluids the characteristic length of self-focusing effects is much shorter than the corresponding to the developement of shock waves [8]. Also, high frequency components are strongly absorbed in such media, so in practice the use of a quasimonochromatic (optical) description for wave propagation is justified.

In a viscous medium sound propagates with a speed $c$ that depends signifficantly on temperature, $c=$ $c_{0}\left(1-\sigma T^{\prime}\right)$, where $c_{0}$ is the speed of sound at some equilibrium (ambient) temperature, $T^{\prime}$ denotes the variation of the medium temperature from that equilibrium due to the intense acoustic wave, and $\sigma$ is the parameter of thermal nonlinearity. The propagation of sound in such a medium has been shown [8, 9] to be described in terms of two coupled equations for pressure, $p^{\prime}$, and temperature, $T^{\prime}$, deviations. These equations have been used to address problems such as self-focusing and selftransparency of sound [8]. They have been also the basis for the analysis of temporal dynamic phenomena in acoustic resonators 10]. In this case, a viscous fluid is bounded by two flat and parallel reflecting surfaces. One of the surfaces, vibrating at a frequency $f$, is an ultrasonic source providing the external forcing. Previous studies on this system [10, 11] have reported, in the frame of the plane-wave approximation, bistability and complex temporal dynamics, in good agreement with the corresponding experiments. In this Letter we extend the previous model considering the effect of sound diffraction 
and temperature diffusion in a large aperture resonator. These effects, which are responsible of the spatial coupling, can play an important role when the Fresnel number of the resonator $\mathcal{F}=l^{2} / \lambda L>>1$ (being $l$ and $L$ its transverse and longitudinal dimensions, respectively).

The intracavity pressure field is decomposed into two counterpropagating traveling waves, $p^{\prime}=p_{+} e^{i(\omega t-k z)}+$ $p_{-} e^{i(\omega t+k z)}+c . c$, where $t$ is time, $z$ is the axial (propagation) coordinate, $\omega=2 \pi f$, and $k=\omega / c_{0}$, whose complex amplitudes $p_{ \pm}$are related through their reflections at the boundaries, and the temperature field is decomposed into a homogeneous component and a grating component, $T^{\prime}=T_{\mathrm{h}}+T_{\mathrm{g}} e^{i 2 k z}+T_{\mathrm{g}}^{*} e^{-i 2 k z}$. All these amplitudes are slowly varying functions of space and time as the fast (acoustical) variations are explicitly taken into account through the complex exponentials. Under the assumption of highly reflecting plates we can adopt a mean field model, where the slowly varying amplitudes do not depend on the axial coordinate $z$ and $p_{-}=p_{+} \equiv p$, ending up with the following dimensionless equations [12]

$$
\begin{aligned}
\tau_{\mathrm{p}} \partial_{\tau} P & =-P+P_{\text {in }}+i \nabla^{2} P+i(H+G-\Delta) P, \\
\partial_{\tau} H & =-H+D \nabla^{2} H+2|P|^{2} \\
\partial_{\tau} G & =-\tau_{\mathrm{g}}^{-1} G+D \nabla^{2} G+|P|^{2} .
\end{aligned}
$$

Here $P=\left(\frac{\sigma \omega t_{\mathrm{p}} t_{\mathrm{h}} \alpha_{0}}{2 \rho_{0}^{2} c_{0} c_{p}}\right)^{1 / 2} p, H=\omega t_{\mathrm{p}} \sigma T_{\mathrm{h}}$, and $G=$ $\omega t_{\mathrm{p}} \sigma T_{\mathrm{g}}$, are new normalized variables, $\tau=t / t_{\mathrm{h}}$ is time measured in units of the relaxation time $t_{\mathrm{h}}$ of the temperature field homogeneous component, and $\tau_{\mathrm{p}}=t_{\mathrm{p}} / t_{\mathrm{h}}$ and $\tau_{\mathrm{g}}=t_{\mathrm{g}} / t_{\mathrm{h}}$ are the normalized relaxation times of the intracavity pressure field and the temperature grating component, respectively. Their original values are given by $t_{\mathrm{p}}^{-1}=c_{0} \mathcal{T} / 2 L+c_{0} \alpha_{0}$, where $\mathcal{T} \ll 1$ is the transmissivity of the plates and $\alpha_{0}$ is the absorption coefficient, and $t_{\mathrm{g}}=$ $1 / 4 k^{2} \chi$, where $\chi=\kappa / \rho_{0} c_{p}$ is the coefficient of thermal diffusivity, $\rho_{0}$ is the equilibrium density of the medium and $\kappa$ and $c_{p}$ are the thermal conductivity and the specific heat of the fluid at constant pressure, respectively. Other parameters are the detuning $\Delta=\left(\omega_{\mathrm{c}}-\omega\right) t_{\mathrm{p}}$, with $\omega_{\mathrm{c}}$ the cavity frequency that lies nearest to the driving frequency $\omega$, and $P_{\text {in }}=\frac{c_{0} t_{\mathrm{p}}}{2 L}\left(\frac{\sigma \omega t_{\mathrm{p}} t_{\mathrm{h}} \alpha_{0}}{2 \rho_{0}^{2} c_{0} c_{p}}\right)^{1 / 2} p_{\text {in }}$, being $p_{\text {in }}$ the injected pressure plane wave amplitude, which we take as real without loss of generality. Finally $\nabla^{2}=\frac{\partial^{2}}{\partial x^{2}}+\frac{\partial^{2}}{\partial y^{2}}$ is the transverse Laplacian operator, where the dimensionless transverse coordinates $(x, y)$ are measured in units of the diffraction length $l_{\mathrm{d}}=c_{0} \sqrt{t_{\mathrm{p}} / 2 \omega}$, and the normalized diffusion coefficient $D=\chi t_{\mathrm{h}} / l_{\mathrm{d}}^{2}$.

The model parameters can be estimated for a typical experimental situation [10]. We consider a resonator with high quality plates $(\mathcal{T}=0.1)$, separated by $L=5 \mathrm{~cm}$, driven at a frequency $f=2 \mathrm{MHz}$, and containing glycerine at $10^{\circ} \mathrm{C}$. Under these conditions the medium parameters are $c_{0}=2 \times 10^{3} \mathrm{~m} \mathrm{~s}^{-1}, \alpha_{0}=10 \mathrm{~m}^{-1}, \rho_{0}=$ $1.2 \times 10^{3} \mathrm{~kg} \mathrm{~m}^{-3}, c_{p}=4 \times 10^{3} \mathrm{~J} \mathrm{~kg}^{-1} \mathrm{~K}^{-1}, \sigma=10^{-2} \mathrm{~K}^{-1}$, and $\kappa=0.5 \mathrm{~W} \mathrm{~m}^{-1} \mathrm{~K}^{-1}\left(\chi=10^{-7} \mathrm{~m}^{2} \mathrm{~s}^{-1}\right)$. In this case $t_{\mathrm{p}}=2 \times 10^{-5} \mathrm{~s}, t_{\mathrm{g}}=6 \times 10^{-2} \mathrm{~s}$, and our length unit $l_{\mathrm{d}}=2 \mathrm{~mm}$. For a resonator with a large Fresnel number, the relaxation of the homogeneous component of the temperature is mainly due to the heat flux through the boundaries, and can be estimated from the Newton's cooling law as $t_{\mathrm{h}} \sim 10^{1} \mathrm{~s}$. (Remind that this is our time unit.) Then the diffusion constant $D \sim 10^{0}$, and the normalized decay times $\tau_{\mathrm{p}} \sim 10^{-6}$, and $\tau_{\mathrm{g}} \sim 10^{-2}$ under usual conditions. We see that the problem is typically very stiff: $0<\tau_{\mathrm{p}} \ll \tau_{\mathrm{g}} \ll 1$. In the following the results will be given to the lowest nontrivial order in these smallest decay times in order to not overburdening the expressions.

The spatially uniform steady state can be obtained by neglecting the derivatives in Eqs. (11). Introducing the notation $W=|P|^{2}, W_{\text {in }}=\left|P_{\text {in }}\right|^{2}$, one has $G=\tau_{\mathrm{g}} W, H=2 W$, and

$$
W_{\text {in }}=W+(\Delta-2 W)^{2} W
$$

The characteristic curve $W$ vs. $W_{\text {in }}$ can display an S-shape, typical of the optical bistability of coherently driven optical Kerr cavities, as we show next. The two turning points of the characteristic $\left(W_{\mathrm{in}, \pm}, W_{ \pm}\right)$, verifying $\mathrm{d} W_{\text {in }} / \mathrm{d} W=0$, and its inflection point $\left(W_{\mathrm{in}, \mathrm{I}}, W_{\mathrm{I}}\right)$, defined by $\mathrm{d}^{2} W_{\text {in }} / \mathrm{d} W^{2}=0$, are given by $W_{ \pm}=$ $\frac{2 \Delta \pm \sqrt{\Delta^{2}-3}}{6}$ and $W_{\mathrm{I}}=\frac{\Delta}{3}$, and the corresponding values of the input pressure follow from Eq. (2). Note that bistability requires $\Delta>\Delta_{0}=\sqrt{3}$, the nascent bistability (NB) occurring at $\Delta=\Delta_{0}$, in which case $W_{ \pm}=W_{\mathrm{I}}=W_{0}=\frac{1}{\sqrt{3}}$. It is very easy to show that this NB inflection point occurs at $P_{\mathrm{in}, 0}=\frac{2}{3^{3 / 4}}$ and that the values of the model variables become $P_{0}=\frac{\sqrt{3}-i}{2 \cdot 3^{1 / 4}}$, $H_{0}=2 W_{0}$, and $G_{0}=\tau_{\mathrm{g}} W_{0}$. Figure 1 shows the behaviour of the homogeneous steady state illustrating the effect of detuning on the character of the solutions [13].

Inspired by previous works on passive optical cavities 14, 15] we shall concentrate our study on conditions close to nascent bistability $\left(\Delta \simeq \Delta_{0}\right)$ and to the vicinity of the inflection point, $\left(P, H, G, P_{\text {in }}\right) \simeq\left(P_{0}, H_{0}, G_{0}, P_{\text {in }, 0}\right)$, where interesting pattern formation properties can be envisaged. Let us just comment before considering in detail the spatiotemporal dynamics of the thermoacoustic resonator that, by focusing on the above conditions we can describe in a consistent way the S-shape of the characteristic curve by using standard multiple scale methods [16].

We consider next the stability of the spatially uniform steady state. We consider perturbations of the form $\exp (i \mathbf{k} \cdot \mathbf{r}+\lambda t)$ and linearize the model Eqs. (1) with respect to them. Further considering the relevant case $0<$ $\tau_{\mathrm{p}} \ll \tau_{\mathrm{g}} \ll 1$, see typical values above, one obtains that, to the leading order, the Lyapunov exponent $\lambda$ can take on the following values: $\lambda \in\left\{-\tau_{\mathrm{p}}^{-1},-\tau_{\mathrm{p}}^{-1},-\tau_{\mathrm{g}}^{-1}, \lambda_{+}\right\}$, 


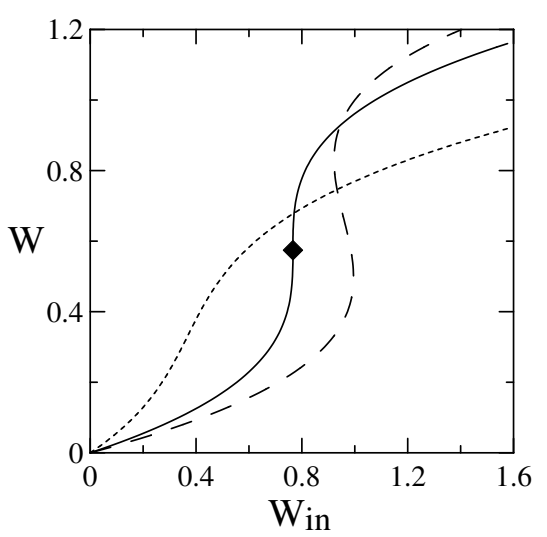

FIG. 1: Characteristic curve output intensity vs input intensity as following from Eq. (2), for three values of the detuning $\Delta$. Monostable regime (dotted line) for $\Delta=1$, nascent bistability (full line) for $\Delta=\sqrt{3}$, and bistable regime (dashed line) for $\Delta=2$ are shown. The symbol marks the position of the inflection point $\left(X_{0}, Y_{0}\right)$ at NB.

where

$$
\lambda_{+}=-1-D k^{2}+4 W \frac{\Delta+k^{2}-2 W}{1+\left(\Delta+k^{2}-2 W\right)^{2}},
$$

$k=|\mathbf{k}|$, and we remind that $W=|P|^{2}$, which fixes the injection pressure through Eq. (2). The obtention of an order parameter equation through a multiple scales analysis requires that a small eigenvalue exists at small wavenumber $k$. In our case $\lambda_{+}$is that eigenvalue. For small $k$ it reads $\lambda_{+}=a+b k^{2}+\mathcal{O}\left(k^{4}\right), a=4 W \frac{\Delta-2 W}{1+(\Delta-2 W)^{2}}-1$, $b=\frac{8 W}{\left[1+(\Delta-2 W)^{2}\right]^{2}}-\frac{4 W}{1+(\Delta-2 W)^{2}}-D$. As we will be concerned with the neighbourhood of the inflection point under NB conditions, let us particularize our analysis to that point. In such case $W=W_{0}, \Delta=\Delta_{0}$ and we obtain $a=0$ and $b=D_{0}-D, D_{0}=\frac{\sqrt{3}}{2}$. Hence, $\lambda_{+}$is actually small for small $k$ in the region of interest. Finally, as we wish to capture pattern formation asymptotically, we must impose that $\lambda_{+}$can change its sign for small $k$. This imposes that the diffusion coefficient $d=D-D_{0} \sim \mathcal{O}(\varepsilon)$, being $\varepsilon$ a small parameter. Additionally, a careful analysis of the homogeneous steady state reveals that, in order to describe consistently the vicinity of the NB point, one must consider the scalings $\delta=\Delta-\Delta_{0} \sim \mathcal{O}\left(\varepsilon^{2}\right)$ and $\mu=\frac{P_{\mathrm{in}}-P_{\mathrm{in}, \mathrm{I}}}{2 \cdot 3^{1 / 4}} \sim \mathcal{O}\left(\varepsilon^{3}\right)$, with $P_{\mathrm{in}, \mathrm{I}}=\frac{2}{3^{3 / 4}}\left(1+\frac{\delta}{2}\right)$. Finally the multiple scale analysis can be performed assuming $p=P-P_{0} \sim \mathcal{O}(\varepsilon), h=H-H_{0} \sim \mathcal{O}(\varepsilon)$ and $g=G-G_{0} \sim \mathcal{O}(\varepsilon)$, together with the slow space and time scales $\tau \sim \mathcal{O}\left(\varepsilon^{-2}\right)$ and $\nabla^{2} \sim \mathcal{O}(\varepsilon)$. Following standard procedures [16], a closed equation for $h$ is obtained as a solvability condition at the third order in $\varepsilon$, which reads

$$
\begin{aligned}
\partial_{\tau} h= & \mu+\frac{\sqrt{3} \delta}{2} h-\frac{3}{4} h^{3}+d \nabla^{2} h-\frac{3}{2} \nabla^{4} h \\
& -\frac{3}{4} h \nabla^{2} h-\frac{3}{2} \nabla^{2} h^{2} .
\end{aligned}
$$

The other fields are related to $h$ as $g=\frac{\tau_{\mathrm{g}}}{2} h$, and $p=\frac{3^{1 / 4}(\sqrt{3}+i)}{4} h$ to the leading order. Equation (44) is a main result of this Letter as it describes the spatiotemporal dynamics of the thermoacoustic resonator in a very simple way. Moreover this equation can be considered as "universal" for our system under typical operating conditions as the numerical values of the coefficients appearing in it are, in the considered limit $\tau_{\mathrm{g}} \rightarrow 0$, very weakly dependent on $\tau_{\mathrm{g}}$ (details will be given elsewhere). Equation (44) is a modified Swift-Hohenberg equation, and has been previously introduced in the optical context for semiconductor lasers 17] and liquid crystal light valve (LCLV) systems [18, 19]. In this context the analysis of Eq. (4) has demonstrated different pattern formation scenarios, including front propagation, pattern competition and localized structures. Experimental evidence has been also reported in [19]. Despite the fundamental differences between the thermoacoustic resonator considered here and the referred optical systems, the derivation of a common order parameter equation allows to extend the optical predictions to the acoustic case.

The homogeneous steady solution $h_{0}$ of Eq. (44) is implicitly given by $\mu=\frac{3}{4} h_{0}^{3}-\frac{\sqrt{3}}{2} \delta h_{0}$, being multivalued when $\delta>0\left(\Delta>\Delta_{0}\right)$, in agreement with the analysis of the microscopic model. In the following we focus on the bistable region, extending between the turning points $h_{0, \pm}= \pm \frac{\sqrt{2 \delta}}{3^{3 / 4}}$. A linear stability analysis shows that, for $d>0$, only the upper branch can be modulationally unstable [18], giving rise to a pattern with wavenumber $k_{\mathrm{c}}^{2}=\frac{15 h_{0}-4 d}{12}$. These results are confirmed by the numerical integration of Eq. (4), performed in a one-dimensional case $\left(\nabla=\partial_{x}^{2}\right)$ which describes, e.g. a physical situation in which the resonator has a slab waveguide configuration confining the sound in the $y$ direction. Figure 2(a) shows the spatiotemporal evolution (from bottom to top) of an initially homogeneous distribution, where the developement of a modulational instability is observed, in agreement with the previous analysis.

In most of the multivalued domain, the extended patterns emerging at the modulational instability are unstable, being a transient state. As shown in Fig. 2(a), neighbour maxima collide and merge, the long-term evolution resulting in a number of localized structures or CSs. In Fig. 2(b) the background and peak values of the CSs (see a profile in the inset) depending on the control parameter $\mu$ are shown with dots. The background amplitude corresponds to the stable uniform state, while the peak value to that of the underlying extended pattern which, as stated in the introduction, is a signature of CSs. Note that CSs are stable and robust structures whenever a pattern and a uniform state coexist.

Figure 2 reveals some important facts. Firstly, localized structures can form spontaneously from the homogeneous steady state, differently from other systems which require a hard local excitation of the medium. Such cir- 

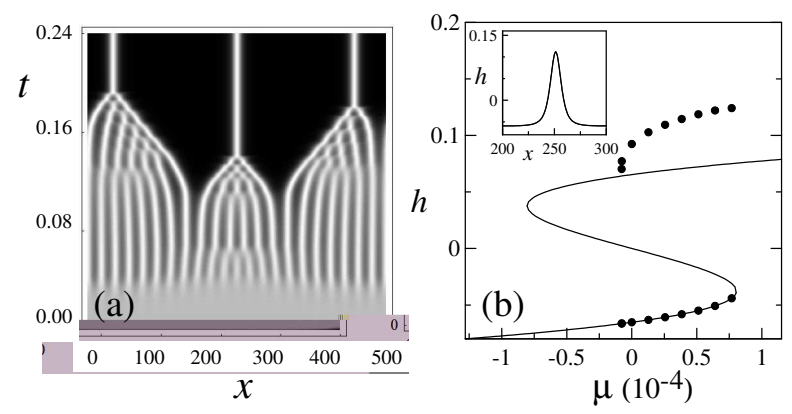

FIG. 2: (a) Numerical simulation of Eq. (4) for $\delta=310^{-3}$. (a) The modulational instability and the further collapse resulting in an array of CSs is shown for $\mu=310^{-5}$. Time runs from bottom to top. (b) Maximum amplitude and background values of the CSs (dots), together with the homogeneous steady solution (full line) depending on the control parameter $\mu$. The inset shows the profile of a CS for parameters in Fig. 2(a). All variables are dimensionless.

cumstance is very relevant in the particular case considered here, where the nature of the driving source (a plane, vibrating rigid surface) makes difficult to implement experimentally a localized excitation. Certainly, the proposal of techniques to address CS in the acoustic resonator will be most relevant.

We have also obtained an analytical, steady CS of Eq. (4),

$$
h(x)=h_{0}+h_{\mathrm{s}} \operatorname{sech}^{2}\left(x / x_{\mathrm{s}}\right),
$$

where the background $h_{0}$ corresponds to the stable homogeneous solution of lower amplitude. The expressions of the peak amplitude $h_{\mathrm{s}}$ and width $x_{\mathrm{s}}$ in (5) are quite involved but can be readily obtained substituting Eq. (5) into Eq.(4). The solution given by Eq. (5) corresponds to an ultrasonic CS but also applies to optical CSs in those systems described by Eq. (41). It is a singular solution, existing for a special value of the injected amplitude $\mu$ determined by the rest of parameters $(d, \delta)$, which is seen to be a particular case of the (non-analytic) CSs family found in the numerical study and illustrated in Fig. 2(b).

Concluding, we have studied the spatiotemporal dynamics of ultrasound in a resonator containing a viscous medium - a thermoacoustic resonator. The microscopic equations have been reduced, close to the nascent bistability point, to a single order parameter equation previously obtained in other contexts. The analysis of the reduced model demonstrates the existence of bistability and modulational instabilities. As a consequence, the system is shown to support cavity solitons, corresponding to states where ultrasound is highly localized in the transverse plane of the resonator. Analytical solutions of such ultrasonic CSs have been obtained. The results reported here will also find application to those systems described by the generalized Swift-Hohenberg equation (4), which is a generic model for pattern formation.

The work was financially supported by the Spanish Ministerio de Educación y Ciencia and the European Union FEDER (Projects FIS2005-07931-C03-01 and -02, and Programa Juan de la Cierva).

[1] M. C. Cross and P. C. Hohenberg, Rev. Mod. Phys. 65, 851 (1993).

[2] A. C. Newell, T. Passot and J. Lega, Ann. Rev. Fluid Mech. 25, 399 (1993).

[3] A. C. Newell and J. V. Moloney, Nonlinear optics (Addison-Wesley, 1992).

[4] P. Mandel, Theoretical problems in cavity nonlinear optics (Cambridge University Press, 1997).

[5] K. Staliunas and V. J. Sánchez-Morcillo, Transverse patterns in nonlinear optical resonators (Springer, 2003).

[6] S. Barland et al., Nature 419, 699 (2002).

[7] F. V. Bunkin, Yu. A. Kravtsov, and G. A. Lyakhov, Sov. Phys. Usp. 29, 607 (1986).

[8] F. V. Bunkin, G. A. Lyakhov, and K. F. Shipilov, Phys. Usp. 38, 1099 (1995).

[9] K. Naugolnykh and L. A. Ostrovsky, Nonlinear wave processes in acoustics (Cambridge University Press, 1998).

[10] G. A. Lyakhov et al., Acoust. Phys. 39, 158 (1993).

[11] V. G. Andreev et al., Sov. Phys. Acoust. 32, 404 (1986).

[12] Details of the derivation will be given elsewhere.

[13] Bistability actually requires simultaneous stability of two solutions, what can occur in our model as we show below. Nevertheless here the important feature is the multivaluedness of the characteristic around the inflection point.

[14] M. Tlidi, M. Georgiou, and P. Mandel, Phys. Rev. A 48, 4605 (1993); M. Tlidi, P. Mandel, and R. Lefever, Phys. Rev. Lett. 73, 640 (1994).

[15] V. J. Sánchez-Morcillo and G. J. de Valcárcel, Quantum Semiclass. Opt. 8, 919 (1996).

[16] A. H. Nayfeh, Perturbation methods (John Wiley \& Sons, New York, 2000)

[17] G. Kozyreff, S. J. Chapman, and M. Tlidi, Phys. Rev. E 68, 015201(R) (2003); G. Kozyreff and M. Tlidi, Phys. Rev. E 69, 066202 (2004).

[18] C. Durniak et al., Phys. Rev. E 72, 026607 (2005).

[19] M. G. Clerc, A. Petrossian, and S. Residori, Phys. Rev. E, 71, 015205 (R) (2005). 